\title{
LOS NIÑOS MENORES DE TRES AÑOS Y LA TELEVISIÓN: Perspectivas de investigación y debate (1999-2010)*
}

\author{
Adriana Rodríguez Sánchez **
}

\author{
CHILDREN UNDER THREE YEARS AND TELEVISION: \\ Perspectives of research and debate (1999-2010)
}

\section{Resumen}

En este texto se reproduce la introducción y las conclusiones de la investigación "Los niños menores de tres años y la Televisión: Perspectivas de investigación y debate (1999-2010)" publicada por el Ministerio de Cultura de Colombia.

En la revisión bibliográfica realizada, se establece que la presencia de la televisión y de otros medios electrónicos es central en la vida cotidiana de los niños, y que el número de horas que pasan frente a la pantalla tiende a incrementarse con el tiempo, con la edad y si tienen televisor en su cuarto. Se encuentra también que aunque buena parte de los videos y programas de televisión dirigidos a niños menores de tres años señalan que persiguen fines educativos, no se ha logrado establecer con claridad que este tipo de productos pueda cumplir con estos propósitos.

En relación con este grupo poblacional le interesa particularmente al Ministerio de Cultura indagaciones que apunten a preguntas tales como: ¿Qué tipo de televisión es pertinente para la infancia?, ¿A qué edades? Además de los contenidos y relatos propios de ésta: ¿Qué otras formas de relación pueden establecer los niños con la mediación de la pantalla?, ¿Qué tan útil es, y especialmente qué tipo de televisión puede ayudar a hacer más digna la vida de la infancia colombiana? pretende además motivar a los investigadores y realizadores colombianos a emprender búsquedas similares, y en especial a usar las investigaciones como criterios al momento de la toma de decisiones en el diseño, producción y emisión de contenidos audiovisuales para niños.

\section{Sumary}

This text reproduces the introduction and conclusions of "Children under three years and Television: Research perspectives and debate (1999-2010)" published by the Ministry of Culture of Colombia.

At the literature review, it is established that the presence of television and other electronic media is central to the daily lives of children. Also, that the number of hours spent in front of the screen tends to increase over time, according to age growth and TV presence in children's room. Although many of the videos and TV shows directed to children under three years indicate the pursue of educational goals, it has not been clearly established whether the products reach these objectives.

In relation to childhood, the Ministry of Culture is particularly interested in questions such as: What kind of television is appropriate for children?, At what ages? In addition to its specific content and stories: What other kind of relationships could be established by children with the mediation of the screen?, How useful is it, and especially, what kind of television can help make life of children more worth in Colombian? The ministry of Culture encourages Colombian researchers and producers to undertake similar quests, and especially invites them to use research criteria into the design, production and broadcasting decisions of audiovisual content for children.

Keywords: Child TV, TV reception and children,

Palabras Clave: Televisión infantil, recepción de la TV y niños.

\footnotetext{
* El Texto completo puede ser consultado en el link del Ministerio de Cultura de Colombia: http://www.mincultura.gov.co/?idcategoria=47761 ** Docente investigadora de la Pontificia Universidad Javeriana- Seccional Cali. Candidata a Doctora en Estudios Científicos Sociales del Instituto Tecnológico y de Estudios Superiores de Occidente-Iteso- de Guadalajara - México. En la actualidad desarrolla su tesis doctoral titulada, provisionalmente: "Procesos de Constitución Social de las Audiencias Televisivas de la Primera infancia: la emergencia de una práctica cotidiana (Cali-Colombia)". Proceso durante el cual ha llevado a cabo una encuesta orientada a caracterizar los contextos de interacción mediática de los niños menores de tres años en la zona urbana de Cali, cuyos resultados corresponden a la primera fase del trabajo de campo. La autora agradece especialmente a los profesores-investigadores Valerio Fuenzalida (Pontificia Universidad Católica de Chile) y Guillermo Orozco Gómez (Universidad de Guadalajara, México) por sus aportes y comentarios al presente documento.

Email: adrianarodriguez@javerianacali.edu.co
} 
Introducción [1]

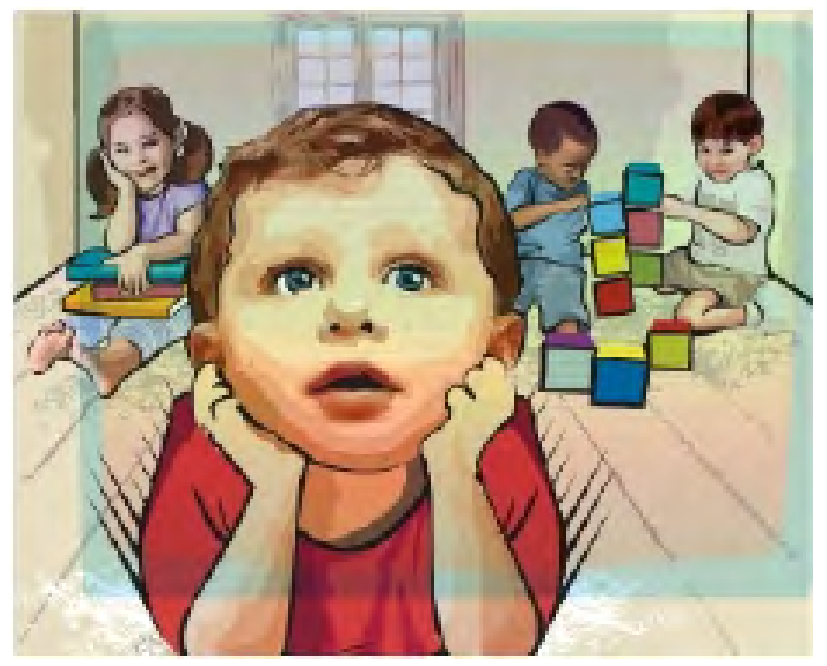

A unque desde los años cincuenta hasta el presente es posible observar cierto desarrollo en las investigaciones sobre la relación de los niños y la televisión (Pecora, Murray y Wartella, 2007), estas se han centrado principalmente en niños en edad escolar $y$ adolescentes (Thakar, Garrinson y Christakis, 2006, p. 2030). Sin embargo, de acuerdo con Wartella, Richert y Robb (2010), los menores de tres años comenzaron a ver televisión en la década de los cincuenta en Estados Unidos, es decir, cuando este medio de comunicación empezó a alcanzar cierta popularidad en los espacios domésticos [2]. Los niños participaban de distinta forma y según sus posibilidades en las rutinas familiares, y una de ellas era ver televisión. Sin embargo, las empresas encargadas de medir la audiencia, como Nielsen, no los registraron en sus cifras.

Por otra parte, la indagación de la academia sobre los niños de estas edades y la televisión ocupó un lugar poco destacado en las agendas de la época. La investigación solo empieza a avanzar en la década de los noventa. No obstante, tal como lo señalan Wartella, Richert y Robb (2010) es posible ubicar algunos estudios que exploraron esta temática antes de ese periodo. Estos autores destacan tres trabajos: el de Schramm, Lyle y Parker (1961), y en los años setenta los de Anderson y Levin (1976) y el de Hollenbeck y Slaby (1979).

En el primero, a partir de una encuesta hecha a finales de los cincuenta, se estableció que el 14\% de los niños de dos años, el $37 \%$ de tres años y el 65\% de cuatro años veían televisión. Según Schramm, Lyle y Parker (1961), los niños a los dos años preguntaban por determinados programas $\mathrm{y}$ a los tres eran casi televidentes regulares. Aunque el objetivo de ese trabajo no era describir exclusivamente lo que ocurría con los niños menores de tres años y la televisión, arroja datos significativos.
La investigación de Anderson y Levin (1976), por su parte, se constituye en el primer estudio publicado sobre la atención a la televisión de los niños entre 12 meses y 4 años, y el primero en considerar la relación entre la atención, los contenidos y las características del programa.

A partir de la observación de un capítulo de Plaza Sésamo, los investigadores establecieron que los niños menores de 36 meses dirigían la mirada hacia la pantalla por periodos muy cortos y preferían hacer otras actividades, y que entre los 24 y 30 meses el nivel de atención se incrementaba. Asimismo notaron cómo la presencia de determinados personajes o sonidos en el programa (una mujer adulta, niños, muñecos, voces peculiares, ritmos o repeticiones) podía contribuir a que el niño fijara su atención en las imágenes televisivas durante más tiempo (Wartella, Richert y Robb, 2010, pp. 118-119).

El trabajo de Hollenbeck y Slaby (1979) examinó las respuestas de niños de seis meses a imágenes y sonidos de la televisión, para ello les fue presentado un programa de cuatro maneras distintas: las imágenes sin audio, solo el audio, las imágenes con audio y un estímulo de control. Este trabajo permitió determinar que las respuestas de los niños eran distintas frente a la estimulación visual y a la sonora. En presencia de imágenes ellos tendían a emitir más sonidos, que cuando solo percibían el audio, sugiriendo que la estimulación sonora de la televisión podía llegar a inhibir la vocalización de los niños, sin embargo, los datos no fueron del todo concluyentes.

En la década de los ochenta, según la revisión hecha por Schmidt et al. (2005), sobresalen dos estudios, el de Anderson, Lorch, Smith y Levin (1981) y el de Meltzoff (1988). El interés del primer trabajo era examinar la relación entre atención y comprensión. Con este propósito, niños de dos, tres, tres años y medio y cuatro años observaron fragmentos de Plaza Sésamo de dos maneras: presentados en secuencias comprensibles y alterando algunos aspectos que los hicieran incomprensibles, como por ejemplo, cambiando el orden de las escenas o que los diálogos de los personajes fueran pronunciados en un idioma extranjero. Los resultados indicaron que cuando el contenido no era comprensible, los niños prestaban menos atención (Schmidt, Bickham, King, Slaby, Branner y Rich, 2005, p. 5).

La investigación de Meltzoff (1988) se centraba en la capacidad de los niños de entender el contenido de la televisión e incorporarlo a su propio mundo, a su conducta (p. 1221). Meltzoff hizo una serie de experimentos en los que niños de 14 y 24 meses observaron en la pantalla un adulto manipulando un juguete, y 24 horas después, les fue entregado esemismojuguete. Según los resultados obtenidos, 
casi todos tendieron a imitar las acciones de los adultos, es decir, pudieron realizar una imitación diferida. Sin embargo, es necesario remarcar que los padres y los experimentadores orientaban la atención de los niños hacia la pantalla y la demostración no iniciaba sino hasta que ellos habían fijado la mirada en la televisión (Richert, Robb y Smith, 2011, p. 86). La interacción con los padres parece haber tenido un rol importante en este estudio.

En los años noventa algunas de las preguntas que acompañaron las investigaciones mencionadas continuaron y emergieron otras, como se verá más adelante. De manera hipotética es posible señalar que el avance de los estudios sobre los niños menores de tres años y la televisión durante esa década se encuentra asociado a tres procesos que podrían estar interrelacionados: una difusión intensiva de los discursos sobre el desarrollo del cerebro en los tres primeros años de vida del ser humano, el rápido crecimiento de la industria audiovisual dirigida a los niños de estas edades y los debates públicos que se han generado en torno a la expansión de estas industrias y sus posibles repercusiones en el desarrollo de los niños.

Durante ese periodo empezaron a difundirse de manera amplia una serie de investigaciones sobre el cerebro [3] en las que se señalaba que las experiencias sensoriales, los cuidados físicos, emocionales y la nutrición que un individuo reciba durante el periodo gestacional y sus tres primeros años de vida resultan definitivos para que se produzca una diferenciación funcional de las células nerviosas y la formación de conexiones entre ellas (Mustard, 2006). De acuerdo con estos hallazgos, los estímulos recibidos por el cerebro.

En estos primeros años determinarían, en buena parte, la calidad de vida de un individuo en su etapa adulta -su aprendizaje, memoria, comportamiento, salud y afectividad- y por extensión a la de la sociedad en general (Mustard, 2006) [4]. La difusión de estas investigaciones contribuyó a que en distintos ámbitos -académicos, políticos, económicos y educativosse incrementara el interés por las condiciones de vida de la población menor de tres años y de la primera infancia [5], término que sirve para designar la franja poblacional que va desde el nacimiento hasta los seis años de edad (Colombia por la primera infancia.

Política pública por los niños y las niñas desde la gestación hasta los 6 años, República de Colombia, 2006, p. 33). En diferentes escenarios, instituciones y organizaciones, tanto internacionales como nacionales, se hicieron llamados frecuentes para cualificar la atención, estímulo y cuidado de los niños de estas edades. Al respecto Unicef en su informe del 2001 señala:
En los primeros momentos, meses y años de vida, cada contacto, cada movimiento y cada emoción en la vida del niño pequeño redunda en una explosiva actividad eléctrica y química en el cerebro, pues miles de millones de células se están organizando en redes que establecen entre ellas billones de sinapsis. Es en esos primeros años de la infancia cuando las experiencias y las interacciones con madres, padres, miembros de la familia y otros adultos influyen sobre la manera en que se desarrolla el cerebro del niño, y tienen consecuencias tan importantes como las de otros factores, entre ellos la nutrición suficiente, la buena salud y el agua pura. Y la manera en que el niño se desarrolla durante este período prepara el terreno para el ulterior éxito en la escuela y el carácter de la adolescencia y la edad adulta (p. 11).

La atención a los niños en los primeros años fue constituyéndose progresivamente para muchos países en un asunto prioritario, en un factor que podía llegar a contribuir, o por el contrario obstaculizar su crecimiento económico y el bienestar social de su población a mediano y largo plazo, como puede observarse en lo planteado por el informe del Banco Mundial, "La promesa del desarrollo en la primera infancia en América Latina y el Caribe" [6], preparado por Vega y Santibáñez (2010).

En medio del reconocimiento de la importancia de una apropiada estimulación de los niños menores de tres años empezaron a proliferar una serie de empresas - o líneas dentro de empresas ya constituidas - orientadas a la elaboración de productos audiovisuales (DVD, CD, videos, videojuegos, software o programas de televisión) destinados a proporcionar a través de distintos recursos, formatos y lenguajes, estímulos adecuados a los niños ubicados en este rango de edad.

Uno de los primeros hitos de estos productos parece haberlo constituido el denominado "efecto Mozart", que promovía la idea de incrementar el potencial intelectual de los niños escuchando de manera frecuente música clásica de determinados compositores (Quart, 2006). Aunque no parecían existir suficientes evidencias científicas que sustentaran esta propuesta, su éxito comercial fue rotundo, e incluso el gobernador de ese entonces de Georgia, en Estados Unidos, propuso utilizar recursos públicos para adquirir y enviar a cada uno de los recién nacidos de su jurisdicción un CD con esta música (Lewin, 2003).

Un segundo hito de estas industrias podría asociarse al lanzamiento del programa de televisión Teletubbies en 1997 [7]. En el 2003, es decir cinco años después de su primera emisión, había sido difundido en 120 países y traducido a 45 idiomas. En 1997 también apareció en el mercado Baby Einstein Company [8], empresa productora de los famosos videos Baby Einstein que tuvieron una rápida acogida y que luego fue adquirida por Walt Disney en el 2001. Durante ese 
mismo periodo, numerosas compañías entraron a participar del floreciente mercado [9], como por ejemplo, Brainy Brand Company (inició labores 1995) [10], Baby Genius (fundada en el 2006) [11], Baby Bumblebee Company (establecida en 1999) [12] Company Oybaby (creada en el 2003) [13] y Abbey Home Media (en el 2000 produjeron Baby Bright) [14].

Un tercer hito parece constituirlo la inauguración de los canales de televisión Baby TV (2003) [15] y BabyFirstTV (2006) [16] que emiten durante los 7 días de la semana 24 horas continuas de programas dirigidos a niños entre 0 y 36 meses.

La aparición de estos productos propició cambios en la relación de los niños más pequeños con lo audiovisual: si antes veían los programas de los otros miembros de la familia, esta vez tendrían a su disposición una oferta variada de videos y televisión diseñada y producida especialmente para ellos.

La difusión y éxito comercial de estos productos audiovisuales ha generado también fuertes debates públicos entre quienes subrayan sus efectos positivos en el desarrollo cognitivo, del lenguaje y afectivo de los niños, y quienes advierten sobre sus peligros: retraso en el lenguaje, alteraciones del sueño, desórdenes de atención, y obesidad, entre otros. Estos debates se han librado especialmente en Estados Unidos y Europa. En algunos países, la discusión ha implicado acciones del Estado.

En Francia, por ejemplo, los canales de televisión que transmiten programas para niños menores de tres años son obligados a emitir el siguiente mensaje: "La TV puede retardar el desarrollo de un niño menor de tres años, aunque se trate de programación que se dirija especialmente a ellos" (Fuenzalida, 2008; Huerta, 2010). O la decisión del Gobierno de Estados Unidos en el 2006 de obligar a la Compañía Walt Disney a retirar de los productos de la línea "Baby Einstein" la etiqueta en la que se los clasificaba como educativos y en el 2009 a devolver el dinero a los compradores que consideraran estos productos inadecuados [17].

Los tres procesos mencionados: la difusión de los discursos del desarrollo del cerebro, la expansión de las industrias audiovisuales especializadas en bebés y los debates públicos sobre su impacto; deben ser entendidos en el marco de procesos más amplios. Tal como lo afirma Livingstone (2009), la presencia del televisor en el hogar y su relación con los niños lleva a considerar dinámicas propias de la globalización, la individualización, la segmentación de los mercados y audiencias, pero también de las transformaciones de la familia, la infancia, las expectativas de los padres con respecto a la crianza, las regulaciones estatales y los adelantos tecnológicos. Tanto los avances de las industrias audiovisuales para niños menores de tres años como la disposición de adquirir estos productos por parte de los agentes educativos encargados de su cuidado (padres y maestros, entre otros), y los debates suscitados, no pueden entenderse por fuera de un complejo entramado social, situado históricamente.

A luz de estos procesos se han planteado las preguntas que guían los estudios sobre la relación entre estos niños y la televisión. Algunas de ellas son: ¿En realidad, qué aprenden los niños de estas edades de la televisión y de los videos? ¿Qué necesitan aprender los bebés de la televisión? ¿Qué pueden ellos aprender? (Krcmar, 2010) ¿Cuáles son las repercusiones de una exposición tan temprana a la televisión? Cada interrogante ha abierto un abanico más amplio de preguntas, que podrían agruparse en algunas tendencias, como podrá observarse más adelante en este documento.

Conclusiones y algunas ideas para próximas investigaciones [18]

A partir de esta revisión se desprenden algunas ideas, preguntas y consideraciones sobre la relación entre los niños menores de tres años y la televisión:

1. De acuerdo con las investigaciones hechas principalmente en Estados Unidos, se ha establecido que la presencia de la televisión y de otros medios electrónicos es central en la vida cotidiana de los niños, y que el número de horas que pasan frente a la pantalla tiende a incrementarse con el tiempo, con la edad y si tienen televisor en su cuarto. A esto se suma el crecimiento de una industria audiovisual dirigida especialmente a ellos. ¿Pero qué ocurre en nuestro contexto específico? ¿Cómo es la presencia de la televisión y de las pantallas en general (videojuegos, computadores, juguetes electrónicos, entre otros) en la vida de los niños menores de tres años, en un país latinoamericano como Colombia? Si se tiene en cuenta que una parte importante de los niños en nuestro país vive en condiciones de pobreza, ¿cómo reciben los mensajes televisivos estos niños en situación de vulnerabilidad?, ¿qué tipo de programas ven?, ¿cuánto tiempo están ellos frente a las imágenes?

Hay algunos datos preliminares, sin embargo aún desconocemos varios aspectos. La relación de los niños menores de tres años con los medios de comunicación parece pasar inadvertida para distintas instancias y la investigación sobre el tema no avanza, o por lo menos no lo hace al ritmo progresivo de la industria y el mercado audiovisual dirigido a ellos. 
2. Aunque buena parte de los videos y programas de televisión dirigidos a niños menores de tres años señalan que persiguen fines educativos, no se ha logrado establecer con claridad que este tipo de productos pueda cumplir con estos propósitos: al respecto hay bastante polémica. Por el contrario, uno de los temas sobre el que existe bastante consenso es el rol que la interacción con los padres $u$ otros agentes educativos tiene en los procesos de aprendizaje de los niños. Más que los productos audiovisuales en sí mismos es la interacción con los agentes educativos lo que marca la diferencia, de tal forma que uno de los desafíos de este tipo de productos es estimular o potenciar la interacción entre los agentes educativos y los niños.

3. Una de las preocupaciones centrales de las investigaciones mencionadas ha sido el tiempo que el niño permanece frente a las imágenes mediáticas, sin embargo, es necesario considerar también el tipo de contenido que observan. Al parecer existe alguna evidencia empírica en la que se señala que cuando los niños mayores de tres años ven programas televisivos con contenido educativo, tienden a presentar a largo plazo mejor desempeño académico y comportamientos pro sociales, lo que no ocurre cuando ven solo programas de entretenimiento con contenidos violentos. ¿Qué tipo de programas de televisión, videos, DVD y videojuegos, ven los niños menores de tres años en Colombia? ¿A qué tipo de contenidos tienen acceso?

4. Las investigaciones revisadas han generado hallazgos interesantes en áreas como el consumo, el aprendizaje y la atención, pero se requiere también indagar y analizar las condiciones económicas, sociales y políticas ligadas con la expansión de los productos audiovisuales dirigidos a niños menores de tres años y su incorporación en la vida cotidiana. La irrupción y saturación de imágenes en el hogar debe interpretarse a la luz de dinámicas sociales amplias, de las lógicas del mercado, de decisiones de orden político y de las condiciones en las que viven las familias de estos niños.

5. Las investigaciones reseñadas han ido tomando distancia progresivamente de modelos reactivos de recepción televisiva en los que se consideraba que los sujetos eran seducidos o atrapados por las características formales de los medios, y cada vez avanzan hacia concepciones de sujetos activos, que procesan información, que orientan sus acciones y las de los otros dependiendo de determinados intereses o motivaciones. El reconocimiento de los niños desde su nacimiento como sujetos partícipes de su aprendizaje (Rogoff, 1993) puede impactar la producción de mensajes acordes con sus capacidades, necesidades y desarrollo.
6. A pesar de los logros de los estudios mencionados, sus resultados tienen algunos límites. Una parte se llevó a cabo en laboratorios y en estos espacios no se logra captar del todo las condiciones habituales en las que un niño y sus padres ven la televisión. En ocasiones es difícil extrapolar o generalizar los resultados de algunos de los experimentos, por el tipo de población que participó en ellos o porque el contenido televisivo comercial no fue considerado en las pruebas Sin embargo, estos trabajos han ido revelando la complejidad de una práctica cotidiana aparentemente simple como es la de ver televisión.

Otro de los límites es que se centran en el hogar, y si bien es cierto que el contacto de los niños con la televisión tiende a iniciarse en casa, ellos también asisten a otros espacios, como guarderías, jardines infantiles y hogares comunitarios, donde los medios de comunicación también pueden estar presentes, por lo que se hace necesario examinar también lo que ocurre en estos lugares.

7. De las investigaciones analizadas surgen varias preguntas abiertas, de las cuales se formula aquí una: ¿Cuáles son las consecuencias a largo plazo en los niños de una exposición tan temprana a los medios de comunicación y en especial a la televisión? Al respecto hay evidencia cruzada.

Por ejemplo, en el trabajo de Pagani et al. (2010), llevado a cabo en Canadá, se encontró que había una relación negativa entre una exposición temprana a la televisión, los logros académicos y hábitos saludables. Luego de hacer un seguimiento a los reportes de padres sobre el tiempo de televisión que veían sus niños con edades que oscilaban entre 29 y 53 meses y comparar los registros escolares de estos en primaria, así como otros indicadores, se señaló por ejemplo, que por cada hora adicional vista a los 29 meses, se notaba un descenso del $6 \%$ en el desempeño en matemáticas y de $13 \%$ de actividad física. En las conclusiones de este estudio se indica que hay riesgos a largo plazo por una exposición temprana a la televisión.

En contraste, los resultados de la investigación de Schmidt et al. (2009), adelantada en Estados Unidos, manifestaba que no se observaba que el tiempo de ver televisión ocasionara ni beneficios ni detrimento de las habilidades cognitivas y del lenguaje de los niños de tres años.

8. El diseño de los productos audiovisuales para estas edades implica tener en cuenta los hitos de desarrollo de estos niños, sus procesos cognitivos, la manera como procesan información de la pantalla, y desde luego su contexto. 
9. Aun en los trabajos analizados no se ha podido establecer con claridad el tipo de competencias, habilidades o conocimientos que puedan ser "enseñados" a los niños a través de la pantalla: ¿Qué pueden aprender los niños de la televisión? ¿Qué se les puede enseñar a través de los medios a los infantes de estas edades? Estas son preguntas que desafían varias instancias: académicas, políticas y desde luego a los productores audiovisuales. Para responderlas es necesario seguir adelantando investigaciones sobre esta área, seguir planteándose interrogantes y considerando los contextos específicos donde los niños menores de tres años crecen en un país latinoamericano como Colombia.

\section{Bibliografía}

American Academy of Pediatric. Council on Communications and Media (2011, November). Media use by children younger than 2 years. Pediatrics, 128(5), 1040-1045. doi: 10.1542/ peds.2011-1753.

American Academy of Pediatrics. Committee on Public Education (1999, August). Media Education. Pediatrics, 104(2), 341-343. doi: 10.1542/peds.104.2.341.

American Academy of Pediatrics. Committee on Public Education. (2001, February). Children, adolescents, and television. Pediatrics, 107(2), 423-426.

Anand, S. \& Krosnick, J. A. (2005, January). Demographic predictors of media use among infants, toddlers, and preschoolers. American Behavioral Scientist, 48(5), 539-561. doi: $10.1177 / 0002764204271512$.

Anderson, D. R. \& Pempek, T. (2005, January). Television and very young children. American Behavioral Scientist, 48(5), 505-522. doi: $10.1177 / 0002764204271506$.

Anderson, D. R., Lorch, E., Smith, R. \& Levin, S. (1981). Effects of peer presence on preschool children's television-viewing behavior. Developmental Psychology, 17(4), 446-453.

Anderson, D. \& Kirkorian, H. (2006). Attention and television. In J. Bryant \& P. Vorderer, Psychology of entertainment (pp. 35-54). New Jersey: Lawrence Erlbaum Associates.

Anderson, D. \& Levin, S. (1976). Young Children's attention to Sesame Street. Child Development, 47(3), 806-811.

Anderson, D., Choi Park, H. \& Lorch Pugzles, E. (1987). Attentional inertia reduces distractibility during young children's tv viewing. Child Development, 58, 798-806.

Anderson, D., Huston, A., Schmitt, K., Linebarger, D., Wright, J. \& Larson, R. (2001). Early childhood television viewing and adolescent behavior: The recontact study. Monographs of the Society for Research in Child Development, 66(1), 1-154.

Arias, N. y Morales, B. (2007). Panorama de los estudios sobre el aprendizaje de palabras en el habla de los niños de 12 meses a 4 años de edad. Folios. Segunda Época(26), 49-63.

Barr, R. (2008). Attention and learning from media during infancy and early childhood. In S. L. Calvert \& B. Wilson, The handbook of children, media and development (pp. 144-165). Malden, MA: Blackwell Publishing.

Barr, R. \& Hayne, H. (1999, October). Developmental changes in imitation from television during infancy. Child Development, 70(5), 1067-1081.

Barr, R. \& Wyss, N. (2008, December). Reenactment of televised content by 2 years olds: Toodlers use language learned from television to solve a difficult imitation problem. Infant Behavior Development, 31(4), 696-703. doi: 10.1016/j.infbeh.2008.04.006.

Barr, R., Danzinger, C., Hilliard, M., Andolina, C. \& Ruskis, J. (2010, June). Amount, content and context of infant media exposure: a parental questionnaire and diary analysis. Interna tional Journal of Early Years Education, 18(2), 107-122. doi: 10.1080/09669760.2010.494431.

Barr, R., Muentener, P. \& García, A. (2007, November). Age-rela $\neg$ ted changes in deferred imitation from television by 6-to 18 monthsolds. Developmental Science, 10(6), 910-921. doi: 10.1111/j.1467-7687.2007.00641.x.

Barr, R., Muentener, P., García, A., Chávez, V. \& Fujimoto, M. (2007, March). The effect of repetition on imitation from television during infancy. Developmental Psychobiology, 49(2), 196-207. doi: 10.1002/dev.20208.

Bronson, P. \& Merryman, A. (2006, September 22). Baby Einstein vs Barbie. Last accesed October 6th, 2011, Available Time: http://www.time.com/ time/nation/article/0,8599,1538507,00.html

Bruer, J. T. (1999). The myth of the first three years. A new understandig of early brain development and lifelong learning. New York: The Free Press.

Carver, L. J., Meltzoff, A. N. \& Dawson, G. (2006, January). Event-related potential (ERP) 
indices of infants' recognition of fa $\neg$ miliar and unfamiliar objects in two and three dimensions. Developmental Science, 9(1), 51-62. doi: 10.1111/j.1467-7687.2005.00463.x.

Certain, L. \& Kahn, R. (2001). Prevalence, correlates and trajectory of television viewing among infants and toddlers. Pe $\neg$ diatrics, 19(4), 634-642. doi: 10.154/peds.109.4.634.

Christakis, D. \& Zimmerman, F. (2009, April). Young children and media: Limitations of current knowledge and future directions for research. American Behavioral Scientist, 52(8), 1177-1185. doi: $10.1177 / 0002764204331540$.

Christakis, D., Garrison, M. \& Zimmerman, F. (2006, October). Television viewing in child care programs: A national survey. Communications Reports, 19(2), 111-120. doi: 10.1080/08934210600920705.

Consejo Nacional de Televisión Chile. (2007). Informe Pre-escolares 0-5 Toons chilenos.

Santiago de Chile: Consejo Nacional de Televisión. Consejo Nacional de Televisión de Chile. (2011, julio). Programación Infantil en Sudamérica 2011. Recuperado octubre 20, 2011, de Consejo Nacional de Chile: http://www.cntv.cl/laprogramacion-infantil-en-sudamerica-2011/ prontus_cntv/2011-07-29/161923.html

DeLoache, J. S. \& Chiong, C. (2009, April). Babies and baby media. American Behavioral Scientist, 52(8), 1115-1135. doi:10.1177/0002764209331537.

DeLoache, J. S., Pierroutsakos, S., Uttal, D., Rosengren, K. \& Gottlieb, A. (1998). Grasping the nature of pictures. Psychological Science, 9, 205-210.

DeLoache, J., Chiong, C., Sherman, K., Islam, N., Vanderborght, M., Troseth, G., et al. (2010). Do babies learn from baby media? Psychological Science, 21(11), 1570-1574. doi: $10.1177 / 0956797610384145$.

Faus Belau, A. (1980). La información televisiva y su tecnología. Pamplona: Universidad de Navarra. Fender, J., Richert, R., Robb, M. \& Wartella, E. A. (2010). Parent teaching focus and toddlers 'learning from an infant DVD. Infant and Child Development, 19, 613-627. doi: 10.1002/ icd.

Fenstermacher, S., Barr, R., Salerno, K., García, A., Shwery, C., Calvert, S., et al. (2010, November). Infant-directed media: An analysis of product information and claim. Infant Child Development, 19, 557-576.doi: 10.1002/icd.718.
Fisch, S. (2000). A capacity model of children's comprehension of educational content on television. Media Psychology, 2(1), 63-91. doi: 10.1207/S1532785XMEPO201_4.

Fuenzalida, V. (2008, 27 de agosto). ¿Prohibir la televisión a los niños? Diario La Tercera, p. 4.

Fuenzalida, V. (2011). Procesos de la audiencia ante al TV. Santiago de Chile: Departamento de Comunicación. Universidad Pontificia Católica de Chile.

Fuenzalida, V. (en prensa). Políticas públicas en ambiente televisivo digital. La oportunidad cultural de la industria de la TV infantil.

Garrison, M. \& Christakis, D. (2005). A teacher in the living room? Educational media for babies, toddlers, and preschoolers. Menlo Park, CA: Kaiser Family Foundation.

Goodrich, S., Pempek, T. \& Calvert, S. (2009, December). Formal production features of infant and toddler DVD. Archives of Pediatrics \& adolescent medicine, 163(12), 1151-1156.

Gregory Thomas, S. (2007). Buy, buy baby: how consumer culture manipulates parents and harms young minds. Estados Uni $\neg$ dos: Mariner.

Gutnick, A. L., Robb, M., Takeuchi, L., \& Kottler, J. (2010, March). Always connected: The new digital media habits of young children. New York: The Joan Ganz Cooney Center at Sesa $\neg$ me Workshop.

Hayne, H., Herbert, J. \& Simcock, G. (2003, June). Imitation from television by 24 and 30 monthsold. Developmental Science, 6(3), 254-261. doi: 10.1111/1467-7687.00281.

Hofer, T., Hauf, P. \& Aschersleben, G. (2007, September). Infants'perception of goaldirected actions on video. British Journal of Developmental Psychology, 25(3), 485-498. doi: 10.1348/026151006X170308.

Hollenbeck, A. R. \& Slaby, R. (1979). Infant visual and vocal responses to television. Child Development, 50(1), 41-45.

Huerta, J. E. (2010). Protection des mineurs in France: A research-based discussion on the effects of television on children less than 3 years of age.

In M. D. Souza \& P. Cabello, The emerging media toddlers (reports Clearinghouse). Göteborg: Nordicom. 
Huston, A. C. \& Wright, J. (1998). Television and the informational and educational needs of children. The ANNALS of The American Academy of Political and Social Science, 557, 9-23.

Khermouch, G. (2004). Brainer babies? Maybe. Big sales? Definitely. Last accesed January 20, 2010, Available Businessweek: http://www. businessweek.com/magazine/content/04_02/ b3865024.htm

Kirkorian, H., Wartella, E., \& Anderson, D. (2008). Media and young children's learning. The Future of Children, 18(1), 39-61. doi: 10.1353/ foc. 0.0002 .

Koolstra, C., \& Lucassen, N. (2004, April). Viewing behavior of children and TV guiadance by parents: A comparison of parents and child reports. Communications, 29(2), 179-198. doi: 10.1515/comm.2004.012.

Kourlaba, G., Kondaki, K., Liarigkovinos, T. \& Manios, Y. (2009, February). Factors associated with television viewing time in toodlers and preschoolers in Greece: The genesis study. Journal of Public Health, 31(2), 222-230 doi: 10.1093/ pubmed/fdp011.

Krcmar, M., Grela, B., \& Lin, K. (2007). Can toodlers learn vocabulary from television? An experimental approach. Media Psychology, 10(1), 41-63. doi: 10.108/15213260701300931.

Krcmar, M. (2010, May). Assessing of Research on Media Cognitive Development, and Infants. Can really Learn from Television and Videos? Journal of Children and Media, 4(2), 119-134. doi:10.1080/17482791003629586.

Kuhl, P., Tsao, F. M. \& Liu, H. M. (2003, July). Foreign-language experience in infancy: Effects of short-term exposure and social interaction on phonetic learning. Proceedings of the National Academy of Sciences of the United States of America, 100(15), 9096-91001. doi: 10.1073/ pnas. 1532872100 .

Lee, S.-J., Bartholic, S. \& Vandewater, E. (2009, March). Predicting Children's Media Use in the USA: Differences in Cross-sectional and Longitudinal Analysis. British Journal Psychological Society, 27(1), 123-143. doi: 10.1348/026151008X401336.

Lewin, T. (2003, October 29). A growing number of video viewers watch from crib. Last accesed September 30, 2011, Available The New York Times: http://www.nytimes.com/2003/10/29/ us/a-growing-number-of-video-viewers-watchfrom-crib.html?src=pm
Linebarger, D., \& Walker, D. (2005, January). Infants'and toddlers' television viewing and languageoutcomes. AmericanBehavioral Scientist, 48(5), 624-645. doi: 10.1177/0002764204271505.

Livingstone, S. (2009). Half a century of television in the lives of our children. The ANNALS of the American Academy of Political and Social Science, 625, 151-163 doi: 10.1177/0002716209338572.

Mandler, J. M. (2005). How to build a baby III: Image-schemas and the transition to verbal thought. Berlin: Mouton de Gruyter.

Meltzoff, A. N. (1988). Imitation of televised models by infants. Child Development, 59, 12211229.

Meyrs, R. G. (2000, enero-abril). Atención y desarrollo de la primera infancia en Latinoamérica y el Caribe. Una revisión de los últimos años y una mirada hacia el futuro. Recuperado septiembre 20, 2009, de Revista Iberoamericana de Educación (Organización de Estados Iberoamericanos): http://www.rieoei.org/rie22a01.htm

Mustard, J. (2006, febrero). Desarrollo de la temprana infancia y del cerebro basado en la experiencia. Bases científicas de la importancia del desarrollo de la primera infancia en un mundo globalizado. Recuperado septiembre 4, 2009, de The Brookings Institution: http://www.brookings. edu/views/papers/200602mustard.htm

Pagani, L., Fitzpatrick, C., Barnett, T. \& Dubow, E. (2010, May). Prospective associations between early childhood television exposure and academic, psychosocial, and physical well-being by middle childhood. Archives of Pediatrics of Adolescent Medicine, 164(5), 425-431.

Pecora, N., Murray, J. \& Wartella, E. (2007). Children and Television. Fifty years of research. New Jersey: Lawrence Erlbaum Associates, Inc. Publishers.

Pempek, T., Kirkorian, H., Richards, J. E., Anderson, D. E., Lund, A. \& Stevens, M. (2010). Video comprehensibility and attention in very young children. Developmental Psychology, 45(5), 1283-1293. doi: 10.1037/a00206614.

Pierroutsakos, S. L. \& Troseth, G. L. (2003, April). Video verite: Infant's manual investigation of objects on video. Infant Behavior and Development, 26(2), 183-199. doi:10.1016/ S0163-6383(03)00016-X .

Quart, A. (2006, July-August). Extreme parenting: Does the baby genius edutainment complex enrich your child's mind or stifle it? Recuperado septiembre 29, 2011, de The Atlantic Magazine (The Atlantic Monthly): http://www.theatlantic. com/magazine/archive/2006/07/extremeparenting/4982/ 
República de Colombia. (2006). Colombia por la primera infancia: política pública por los niños y niñas, desde la gestación hasta los 6 años.

Rice, M. L., Huston, A., Truglio, R. \& Wright, J. (1990, May).Words from "Sesame Street" Learning Vocabulary while Viewing. Developmental Psychology, 26(3), 421-428.

Richert, R. A., Robb, M. \& Smith, E. (2011, January-February). Media as social partners: Children's learning from screen media. Child Development, 82(1), 82-95. doi: 10.1111/j14678624.2010.01542.x

Richert, R., Robb, M., Fender, J., \& Wartella, E. (2010, May). Word learning from baby videos. Archives of Pediatrics and Adolescent Medicine, 164(5), 432-437.doi:10.1001/archpediatrics.2010.24.

Rideout, V. (2007). Parents, children and media: A Kaiser Family Foundation Survey. Menlo Park, CA: Kaiser Family Foundation.

Rideout, V. J., Vandewater, E. A. \& Wartella, E. A. (2003). Zero to six: Elextronic media in the lives of infants, toddlers and preschoolers. Menlo Park CA: The Henry J. Family Kaiser Foundation.

Rideout, V. \& Hamel, E. (2006, May). The media family: Electronic media in the lives of infants, toddlers, preschoolers and their parents. Menlo Park, CA: Kaiser Family Foundation.

Robb, M., Richert, R. \& Wartella, E. (2009, March). Just a talking book? Word learning from watching baby videos. British Journal of Developmental Psychology, 27(1), 27-45. doi: $10.1348 / 026151008 X 320156$.

Rodríguez, A., Rodríguez, R. y Sevilla, M. (2006, enero-junio). Biografía pública de la televisión en Cali, según información de prensa (1954-1970). Signo y Pensamiento, XXV(48), 55-75.

Rodríguez, A., Rodríguez, R. y Sevilla, M. (2008, enero-junio). Más televisores que televisión: espacios domésticos y la televisión en Cali 19541970. Signo y Pensamiento, XVII(52), 145-164.

Rogoff, B. (1993). Aprendices del pensamiento. El desarrollo cognitivo en el contexto social. Barcelona: Ediciones Paidós.

Roseberry, S., Hirsh-Pasek, K., Parish-Morris, J. \& Michnick, R. (2009). Live action: Can young children learn verbs from video? Child Development, 80(5), 1360-1375. doi: 10.1111/j.1467-8624.2009.01338.x.

Roskos-Ewoldsen, D. R. \& Roskos-Ewoldsen, B. (2010). Message processing. In C. Berger, M.
Roloff \& D. Roskos-Ewoldsen, The handbook of Communication Science (pp. 129-145). Los Angeles: Sage Publications.

Ruff, H. \& Rothbart, M. K. (1996). Attention in early development: Themes and variations. New York: Oxfor University Press.

Schmidt, M. E., Bickham, D., King, B., Slaby, R., Branner, A. \& Rich, M. (2005). The effects of electronic media on children ages zero to six: A history of research. Henry J. Kaiser Family Foundation-Center on Media and Child Health. California: Kaiser Family Foundation.

Schmidt, M. E., Rich, M., Rifas-Shiman, S., Oken, E. \& Taveras, E. (2009, March). Television viewing in infancy and child cognition at 3 years of age in a US cohort. Pediatrics, 123(3), 370-375. doi: 10.1542/peds.2008-3221.

Schmitt, K. \& Anderson, D. (2002). Television and reality: Toddlers' use of visual information from video to guide behavior. Media Psychology, 4(1), 51-76.

Schramm, W., Lyle, J. \& Parker, E. (1961). Television in the lives of our children. Standford CA: Standford University Press.

Strouse, G. \& Troseth, G. (2008, December). "Don't try this at home": Toddlers' imitation of new skills from people on video. Journal of Experimental Child Psychology, 101 (4), 262-280. doi: 10.1016/j.jecp.2008.05.010.

Thakar, R., Garrinson, M. \& Christakis, D. (2006, November). A sistematic review for effects of television viewing by infants and preschoolers. Pediatrics, 118(5), 2025-2031. doi:10.1542/ peds.2006-1307.

Troseth, G. \& DeLoache, J. (1998, August). The medium can obscure the message: Young children' understanding of video. Child Development, 69(4), 950-965.

Troseth, G., Saylor, M. \& Archer, A. (2006, May-June). Young children's use of video as a source of socially relevant information. Child Development, 77(3), 786-799.

Unicef (2001). Estado mundial de la infancia. New York: Fondo de las Naciones Unidas para la Infancia.

Vandewater, E. \& Lee, S. J. (2009, April). Measuring children's media use in the digital age: Issues and challenges. American Behavioral Scientist, 52(8), 1152-1176. doi: 10.1177/0002764209331539. 
Vandewater, E., Rideout, V., Wartella, E., Xuan, H., Lee, J. \& Shim, M.-S. (2007, May). Digital childhood: Electronic media and technology use among infants, toddlers, and preschoolers. Pediatrics, 119(5), 1006-1015. doi: 10.1542/ peds.2006-1804.

Vega, E. y Santibáñez, L. (2010). La promesa del desarrollo de la primera infancia en América Latina y el Caribe. Banco Mundial. Washington: Ediciones Mayol

Wartella, E. A. \& Robb, M. (2008). Historical and recurring concerns about children's use of the mass media. In S. L. Calvert \& B. Wilson, The handbook of children, media and development (pp. 7-26). Malden MA: Blackwell Publishing Ltd.

Wartella, E., Richert, R. \& Robb, M. (2010, June). Babies, television and video: How did we get here? Developmental Review, 30(2), 116-127. doi: 10.1016/j.dr.2010.03.008.

Zack, E., Barr, R., Gerhardstein, P., Dickerson, K. \& Meltzoff, A. (2009). Infant imitation from television using novel touch screen technology. British Journal of Developmental Psychology, 27, 13-26. doi:10.1348/026151008X334700.

Zimmerman, F., Christakis, D. A., \& Meltzoff, A. (2007, May). Television and DVD/Video viewing in children younger than 2 years old. Archives of Pediatrics \& Adolescent Medicine, 161, 473-479.

\section{Notas}

[1] Rodriguez, A. (2012). Los niños menores de tres años y la televisión. Perspectivas de investigación y debate (1999-2010). Bogotá: Ministerio de Cultura - Dirección de Comunicaciones. (pp. 9 18) . Recuperado en: http://www.mincultura.gov. co/ idcategoria $=47761$.

[2] En otros países, este proceso ocurriría en momentos posteriores y dependiendo de las condiciones sociales, culturales políticas y económicas que posibilitaron $\mathrm{u}$ obstaculizaron la incorporación tanto del aparato de recepción, como dela programación televisiva en los espacios, tiempos y rutinas hogareñas. Para observar el proceso diferenciado, y en algunos casos similar, de instalación y difusión de la televisión en el mundo, puede consultarse el estudio comparativo de Faus Belau (1980), y para el caso de una ciudad colombiana como Cali los trabajos de Rodríguez, Rodríguez y Sevilla (2008; 2006).

[3] En 1997, se llevó a cabo un evento que contribuyó significativamente a difundir estas investigaciones; la conferencia "Early Childhood Development and Learning: What New Research on the Brain Tell Us About Our Youngest Children", llevada a cabo en la Casa Blanca durante el Gobierno de Clinton. A ella fueron invitados, como expositores, numerosos expertos en neurobiología y en el desarrollo de la primera infancia para explicar el alcance del desarrollo del cerebro en los tres primeros años de vida y su impacto en las etapas posteriores. Uno de los objetivos de la conferencia era mostrar tanto al Congreso como al público la importancia de disponer de fondos para financiar programas destinados al cuidado y desarrollo de la primera infancia (Gregory Thomas, 2007, p. 35).

[4] Los discursos sobre el desarrollo del cerebro en los tres primeros años de vida no están exentos de debate y en algunas ocasiones se los examina como mitos que pueden llegar a obstaculizar la comprensión del funcionamiento de este órgano en el transcurso de la vida de un individuo. Ver por ejemplo: Bruer (1999).

[5] La idea de la atención y desarrollo de la primera infancia y la de la educación inicial surgen en 1990 en el marco de la Conferencia Mundial de Educación para Todos, celebrada en Jomtiem, en la que se planteaba: «El aprendizaje comienza al nacer. Esto requiere atención y desarrollo de la primera infancia. Ésta puede proporcionarse implicando a las familias, a las comunidades o a programas institucionales, según corresponda» (Meyrs, 2000, p. 1).

[6] En este informe se señala que en algunos países latinoamericanos aproximadamente $40 \%$ de los niños menores de cinco años son pobres.

La precariedad de la atención y nutrición a la que están sometidos estos niños puede convertirse en un factor definitivo para reproducir e incrementar la pobreza en los próximos años. Por esta razón, el Banco Mundial insta a los distintos Gobiernos latinoamericanos y del Caribe a invertir en esta población, con el convencimiento de las repercusiones positivas que ello puede ofrecer.

[7] Al respecto: www.ragdoll.co.uk. La serie dejó de ser producida en 1999 después de 365 capítulos, pero su difusión continuó a través de $\mathrm{BBC}$ y posteriormente fue diseñada otra versión de Teletubbies.

[8] http://www.babyeinstein.com/en/our_story/ history/

[9] Para el 2004, las ventas anuales de videos para bebés había alcanzado 100 millones de dólares (Khermouch, 2004) y en el 2005, 200 millones (Bronson y Merryman, 2006).

[10] http://thebrainybrandscompany.com/ 
[11] Baby Genius es una marca registrada de Pacific Entertainment Corporation http://www. babygenius.com/investors-company-description. html

\section{[12] http://www.babybumblebee.com}

[13] http://www.oybaby.com/blogs/press/852102original-oybaby-company-launch-press-releasenovember-2003

[14]http://www.abbeyhomemedia.com/html/ index.asp

[15] En diciembre del 2007 Baby TV firmó 19 nuevos contratos de retransmisión en Latinoamérica, incrementando la base de abonados a más de un millón en esta región. "Los acuerdos fueron negociados a través de Fox Latin American Channels y entrarán en vigencia a partir de diciembre de 2007 y enero de 2008. Estos nuevos convenios incluyen a VTR en Chile; Cablevisión, PCTV y Maxcom en Méico; TV Cable en Bogotá; UNE y Codisert en Colombia; Intercable y Supercable en Venezuela; CableOnda en Panamá; Telecable Nacional Caucana, Wind Telecom y Telecable del Nordeste en República Dominicana; TDS Systems en Curazao; Claro TV y Z\&L en América Central y SMC Comunicaciones en el Caribe. Estos nuevos contratos adhieren a otros que incluyen a Cablevisión y Multicanal en Argentina; Nuevo Siglo en Uruguay; CTV Telecom en Colombia; Multivisión en Bolivia; Prosat en Guatemala; CableTica en Costa Rica y Estesa en Nicaragua, entre otros". http://www.babytvchannel.com/es/ view_article.aspx?l=1\&i=61\&si=44

[16] http://www.babyfirsttv.com/

[17] Los reclamos contra la línea Baby Einstein fueron liderados por Campaign for a Commercial Free Childhood ante Federal Trade Commission. Ver recopilación de los argumentos utilizados en el debate: http://www.commercialfreechildhood. org

[18] Rodriguez, A. (2012). Los niños menores de tres años y la televisión. Perspectivas de investigación y debate (1999-2010). Bogotá: Ministerio de Cultura - Dirección de Comunicaciones. Conclusiones (pp. 61 - 65). Recuperado en: http://www.mincultura.gov. $\mathrm{co} /$ ? idcategoria $=47761$.

\section{Para citar este artículo:}

Rodríguez, A. (2012). Los niños menores de tres años y la televisión. Perspectivas de investigación y debate (1999-2010). Bogotá: Ministerio de Cultura - Dirección de Comunicaciones. Recuperado de: http://www.mincultura.gov.co/?idcategoria=47761

Apartados publicados en: Revista Luciérnaga Año 4, N7. Grupo de Investigación en Comunicación, Facultad de Comunicación Audiovisual, Politécnico Colombiano Jaime Isaza Cadavid. Medellín-Colombia. ISSN 2017-1557. Págs. 12-22.

DOI. 10.33571/revistaluciernaga.v4n7a2 